\title{
Dramatic Response with Single-Agent Ibrutinib in Multiply Relapsed Marginal Zone Lymphoma with MYD88 ${ }^{\text {L265P }}$ Mutation
}

\author{
Ryan C. Lynch Ranjana H. Advani \\ Division of Oncology, Department of Medicine, Stanford University School of Medicine, \\ Stanford, CA, USA
}

\section{Keywords}

Marginal zone lymphoma · Targeted therapy ·

\begin{abstract}
The B-cell receptor signaling pathway is important in the lymphomagenesis of many lymphomas, including marginal zone lymphoma (MZL). Herein we describe a case of extranodal MZL refractory to multiple lines of therapy. The presence of an IgM paraprotein prompted further evaluation, and the patient was found to have an MYD88 ${ }^{\mathrm{L} 265 \mathrm{P}}$ mutation. Treatment with ibrutinib led to a dramatic response with prompt resolution of symptoms and significant improvement in measurable sites of disease. The excellent response to ibrutinib in our patient with MYD8 $8^{L 265 P}$-mutated refractory MZL supports a biological rationale for its use.
\end{abstract}

(C) 2017 The Author(s)

Published by S. Karger AG, Basel

\section{Introduction}

Marginal zone lymphoma (MZL) is a heterogeneous group of indolent lymphoproliferative disorders that account for $\sim 5 \%$ of all cases of non-Hodgkin lymphoma (NHL) in adults [1]. Immunohistochemistry is typically positive for B-cell markers including CD19, CD20, 
and CD22, and negative for CD5, CD10, and CD23 [2]. MZL can also be associated with a monoclonal paraprotein, most commonly IgM ( $\sim 29 \%$ of cases) $[3,4]$.

MYD88 is an adaptor protein of the Toll-like receptor that participates in the innate immune response and plays a key role in B-cell homeostasis [5]. Recently, MYD88 ${ }^{\mathrm{L} 265 \mathrm{P}}$ mutation has been reported in $\sim 90 \%$ of lymphoplasmacytic lymphomas (LPL)/Waldenström macroglobulinemia (WM) cases, and helps in distinguishing LPL/WM from other similar small B-cell NHL [6]. MYD88 ${ }^{\mathrm{L} 265 \mathrm{P}}$ mutation leads to constitutive activation of IRAK and Bruton tyrosine kinase (BTK)-mediated signaling, which increases proliferation of the affected mature B lymphocytes through NFKB and JAK/STAT3 signaling $[6,7,8]$. Ibrutinib, a BTK inhibitor, leads to decreased binding of mutant MYD88 protein to BTK, resulting in reduced downstream NFKB signaling and increased apoptosis in WM cell lines [7]. Ibrutinib has considerable clinical activity in several B-cell neoplasms including WM [9], which led to its FDA approval for that indication [10]. In fact, the 2016 revision of the WHO classification recommends MYD88 mutation analysis in patients with newly diagnosed LPL, particularly those with concurrent IgM monoclonal gammopathy, given its importance in optimizing therapy [11]. MYD88 ${ }^{\mathrm{L} 265 \mathrm{P}}$ is not specific to WM, but data on its frequency in other subtypes of indolent B-cell NHL is limited. Two small series report the mutation in 4-21\% of MZL cases [12, 13]. Interestingly, those with MYD88 ${ }^{\mathrm{L} 265 \mathrm{P}}$ were also more likely to present with an IgM paraprotein [13].

\section{Case Description}

Herein we describe a case of extranodal MZL refractory to multiple lines of therapy. The presence of an IgM paraprotein prompted further evaluation, and the patient was found to have an MYD88 ${ }^{\mathrm{L} 265 \mathrm{P}}$ mutation. Treatment with ibrutinib led to a dramatic response with prompt resolution of symptoms and significant improvement in measurable sites of disease.

\section{Case Presentation}

A 66-year-old male first presented 11 years ago with fatigue, fever, shortness of breath, generalized adenopathy, and mild bilateral proptosis. Staging computed tomography (CT) showed extensive lymphadenopathy above and below the diaphragm and large bilateral pleural effusions. Excisional biopsy of a right axillary lymph node showed effacement of the normal nodal architecture with a neoplastic lymphoid infiltrate composed of small- to medium-sized lymphocytes consistent with MZL. Flow cytometry demonstrated a clonal B-cell population that expressed bright monoclonal kappa, CD19, CD20, and CD45, and was negative for CD5 and CD10. Pleural fluid from the left lung and bone marrow were also involved. Orbital MRI showed diffuse signal abnormality involving the extraocular musculature, consistent with diffuse lymphomatous infiltration. Laboratory data were notable for a normal complete blood count and LDH, and monoclonal IgM kappa protein of $1.0 \mathrm{~g} / \mathrm{dL}$.

The patient was started on treatment with R-CVP (rituximab, cyclophosphamide, vincristine, and prednisone). Restaging CT after 2 cycles of therapy showed no response, hence treatment was intensified to the anthracycline-based regimen R-CHOP (rituximab, cyclophosphamide, doxorubicin, vincristine, and prednisone) for 2 cycles without any radiographic improvement. Given the poor response to the latter, CVP was reinitiated with a modification of cyclophosphamide to an oral formulation at $400 \mathrm{mg} / \mathrm{m}^{2}$ on days $1-5$. Rituximab 
was omitted due to prior hypersensitivity reactions. With this modified regimen the patient had significant improvement and achieved a complete response (CR) after 4 cycles. Six years later, he developed fatigue, anemia, and recurrent cervical, axillary, mediastinal, retroperitoneal, and inguinal lymphadenopathy. Bone marrow biopsy revealed $>50 \%$ involvement with MZL. He was treated with 6 cycles of rituximab and bendamustine (rituximab omitted for the last 4 cycles due to recurrent hypersensitivity reaction) and achieved a partial remission (PR) and normalization of hemoglobin.

His disease remained well controlled for $\sim 2$ years when he presented with increasing fatigue and mild anemia (hemoglobin $10.5 \mathrm{~g} / \mathrm{dL}$ ). Repeat bone marrow biopsy showed $>20 \%$ involvement with MZL. A CT scan showed interval worsening of known sites of involvement above and below the diaphragm as well as abnormal enhancement in the bilateral orbital fat consistent with lymphomatous involvement. Treatment was started with the previously used modified CVP regimen for 6 cycles followed by palliative radiotherapy to the bilateral orbits (4 Gy) with a PR. Six months later, he presented with right lower extremity pain and foot drop. A CT scan showed interval worsening of known lymphadenopathy, and MRI of the lumbar spine showed diffuse thickening of the cauda equina nerve roots suggestive of involvement. Treatment with obinutuzumab was initiated without any response. Laboratory data at this point were notable for hemoglobin of $8.7 \mathrm{~g} / \mathrm{dL}$ and an elevated serum IgM kappa monoclonal protein at 2,450 mg/dL. Since MYD88 mutations had recently been described in a subset of MZL with IgM paraprotein [12], we evaluated his bone marrow aspirate, which revealed an MYD88 ${ }^{\mathrm{L} 265 \mathrm{P}}$ mutation.

Given the lack of standard chemotherapy options and the impressive track record of ibrutinib in MYD88-mutated WM, we initiated therapy at $420 \mathrm{mg} /$ day. Within a day he had rapid improvement in systemic symptoms, which completely resolved over the next few weeks. At the same time, significant improvement was also seen in hemoglobin and serum IgM levels (Fig. 1). CT imaging showed improvement in all sites of involvement (Fig. 2). At the time of this report the patient has been on treatment for 14 months with ongoing response.

\section{Discussion}

B-cell receptor (BCR) signaling has emerged as the driving pathway of lymphoma development in many mature B-cell lymphomas [14]. While its exact role in the development of MZL is unknown, it is postulated that chronic infection (e.g., Helicobacter pylori, hepatitis C) may lead to antigen-mediated BCR signaling activation and eventual lymphomagenesis $[15$, 16]. Studies suggest that MYD88 ${ }^{\mathrm{L} 265 \mathrm{P}}$ plays a key role in the pathogenesis of IgM monoclonal gammopathy-related diseases [17], and it is plausible that it may be an additional mechanism of BCR activation in MZL.

Recently, ibrutinib has been approved for relapsed/refractory MZL on the basis of a single-arm phase II study that treated 63 patients at $560 \mathrm{mg}$ daily [18]. Independent assessment reported an overall response rate of $48 \%$ (2 CR and 27 PR) and a median progressionfree survival of 18 months. To the best of our knowledge the latter study did not include data on IgM levels or MYD88L265P mutation status. The excellent response to ibrutinib in our patient supports a biological rationale for its use in MYD88L265P-mutated refractory MZL. With better understanding of lymphoma biology in an era of precision medicine, it is increasingly important to match a patient's molecular profile with novel therapies with the goal of individualizing treatment. Given the prompt response seen in our patient, it is reasonable to 
consider testing for MYD88 ${ }^{\mathrm{L} 265 \mathrm{P}}$ in cases of MZL with IgM paraprotein as it may identify patients with a higher likelihood of a robust response compared to patients without the mutation.

Resistance to ibrutinib has been reported in WM patients who lack MYD88L265P, and the response is less robust in patients with concurrent MYD88 ${ }^{\mathrm{L} 265 \mathrm{P}}$ and CXCR4WHIM mutations [9]. A small study suggests that the incidence of CXCR4 WHIM mutations in WM and MZL patients with MYD88L265P mutations was not significantly different [17]. However, the latter needs confirmation in larger data sets of MZL before it can be adopted into clinical practice.

In conclusion, our report lends support for a biological rationale for treating MZL with ibrutinib and consideration for MYD88 ${ }^{\text {L265P }}$ testing in cases associated with an IgM paraprotein.

\section{Statement of Ethics}

The authors certify that the material in this case report has been acquired according to modern ethical standards and has been presented in such a fashion as to protect the rights of the patient involved.

\section{Disclosure Statement}

R.H.A. has received honorarium for advisory board as well as institutional research funding from Pharmacyclics. R.C.L. has no relevant disclosures to declare.

\section{Author Contributions}

Both R.H.A. and R.C.L. participated in the care of the patient, and both authors prepared the manuscript.

\section{References}

1 Armitage JO, Weisenburger DD: New approach to classifying non-Hodgkin's lymphomas: clinical features of the major histologic subtypes. Non-Hodgkin's Lymphoma Classification Project. J Clin Oncol 1998;16:2780-2795.

2 Swerdlow SH, Campo E, Harris NL, et al: WHO Classification of Tumours of Haematopoietic and Lymphoid Tissues, ed 4. Geneva, World Health Organization, 2008.

-3 Martinez-Lopez A, Curiel-Olmo S, Mollejo M, et al: MYD88 (L265P) somatic mutation in marginal zone B-cell lymphoma. Am J Surg Pathol 2015;39:644-651.

-4 Berger F, Traverse-Glehen A, Felman P, et al: Clinicopathologic features of Waldenström's macroglobulinemia and marginal zone lymphoma: are they distinct or the same entity? Clin Lymphoma 2005;5:220-224.

5 Treon SP, Cao Y, Xu L, et al: Somatic mutations in MYD88 and CXCR4 are determinants of clinical presentation and overall survival in Waldenström macroglobulinemia. Blood 2014;123:2791-2796.

6 Treon SP, Xu L, Yang G, et al: MYD88 L265P somatic mutation in Waldenström's macroglobulinemia. N Engl J Med 2012;367:826-833.

7 Yang G, Zhou Y, Liu X, et al: A mutation in MYD88 (L265P) supports the survival of lymphoplasmacytic cells by activation of Bruton tyrosine kinase in Waldenström macroglobulinemia. Blood 2013;122:1222-1232.

-8 Ngo VN, Young RM, Schmitz R, et al: Oncogenically active MYD88 mutations in human lymphoma. Nature 2011;470:115-119. 
-9 Treon SP, Tripsas CK, Meid K, et al: Ibrutinib in previously treated Waldenström's macroglobulinemia. N Engl J Med 2015;372:1430-1440.

10 Raedler LA: Imbruvica (ibrutinib): first drug approved for the treatment of patients with Waldenström's macroglobulinemia. Am Health Drug Benefits 2016;9:89-92.

-11 Swerdlow SH, Campo E, Pileri SA, et al: The 2016 revision of the World Health Organization classification of lymphoid neoplasms. Blood 2016;127:2375-2390.

12 Insuasti-Beltran G, Gale JM, Wilson CS, et al: Significance of MYD88 L265P mutation status in the subclassification of low-grade B-cell lymphoma/leukemia. Arch Pathol Lab Med 2015;139:1035-1041.

13 Hamadeh F, MacNamara SP, Aguilera NS, et al: MYD88 L265P mutation analysis helps define nodal lymphoplasmacytic lymphoma. Mod Pathol 2015;28:564-574.

14 Niemann CU, Wiestner A: B-cell receptor signaling as a driver of lymphoma development and evolution. Semin Cancer Biol 2013;23:410-421.

15 Quinn ER, Chan CH, Hadlock KG, et al: The B-cell receptor of a hepatitis C virus (HCV)-associated nonHodgkin lymphoma binds the viral E2 envelope protein, implicating HCV in lymphomagenesis. Blood 2001;98:3745-3749.

16 Suarez F, Lortholary 0, Hermine 0, et al: Infection-associated lymphomas derived from marginal zone B cells: a model of antigen-driven lymphoproliferation. Blood 2006;107:3034-3044.

17 Cao XX, Meng Q, Cai H, et al: Detection of MYD88 L265P and WHIM-like CXCR4 mutation in patients with IgM monoclonal gammopathy related disease. Ann Hematol 2017;96:971-976.

18 Noy A, de Vos S, Thieblemont C, et al: Targeting BTK with ibrutinib in relapsed/refractory marginal zone lymphoma. Blood 2017;129:2224-2232.

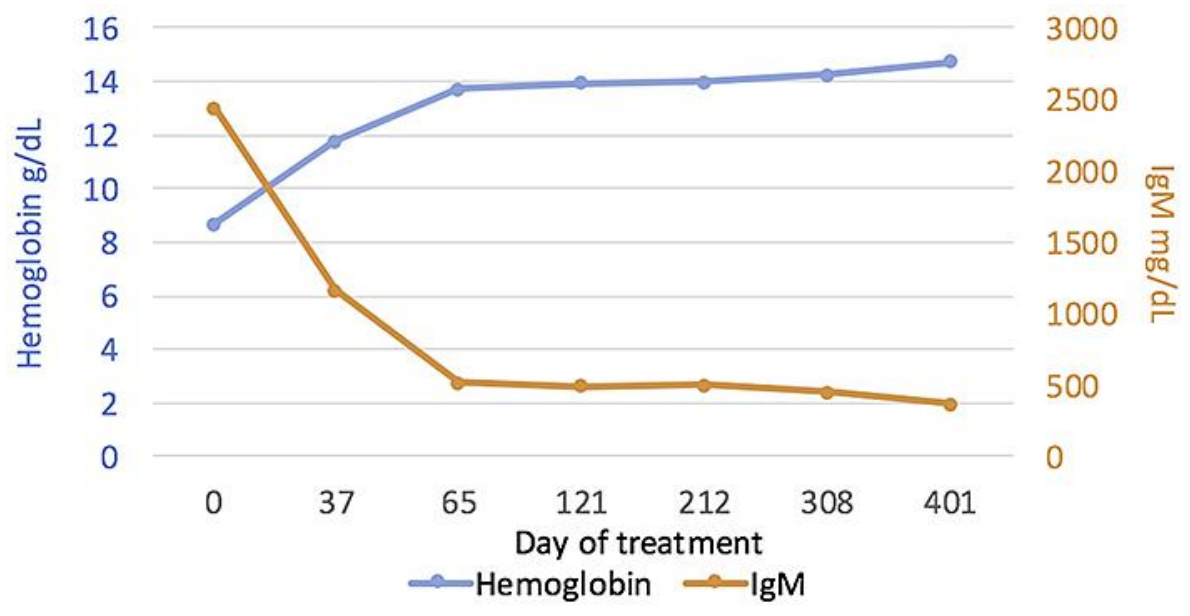

Fig. 1. Improvement in hemoglobin and IgM with ibrutinib treatment. 


\section{Case Reports in Oncology}

\begin{tabular}{l|l} 
DOI: $10.1159 / 000480292$ & $\odot 2017$ The Author(s). Published by S. Karger AG, Basel \\
\hline
\end{tabular} www.karger.com/cro

Lynch and Advani: Dramatic Response with Single-Agent Ibrutinib in Multiply Relapsed Marginal Zone Lymphoma with MYD88 ${ }^{1265 P}$ Mutation
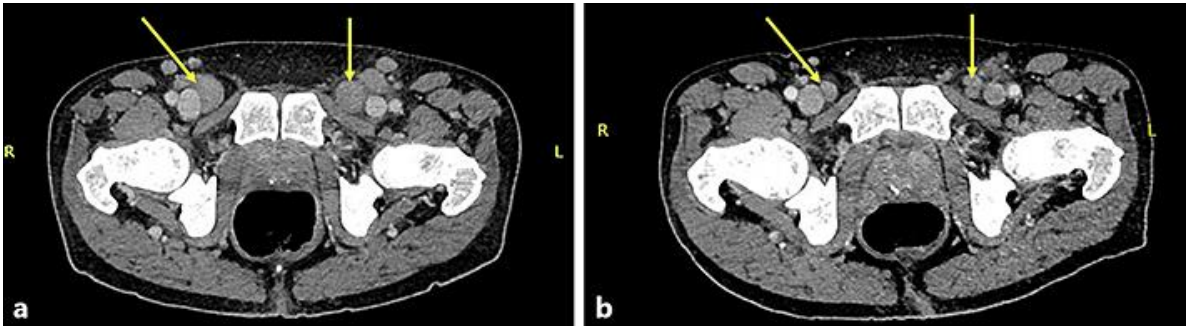

Fig. 2. CT scan of bilateral inguinal lymphadenopathy before (a) and 201 days after (b) initiation of ibrutinib. 